Quim. Nova, Vol. 35, No. 5, 1030-1035, 2012

\title{
DESENVOLVIMENTO E VALIDAÇÃO DE MÉTODO PARA O DOSEAMENTO DE TIBOLONA EM CÁPSULAS POR CROMATOGRAFIA LÍQUIDA DE ALTA EFICIÊNCIA
}

\author{
Hernandez Bozardi da Rosa e Renata Cristine Tolotti \\ Centro de Ciências da Saúde, Universidade de Caxias do Sul, Rua Francisco Getúlio Vargas, 1130, 95070-560 Caxias do Sul - \\ RS, Brasil \\ Diogo dos Santos Miron \\ Curso de Farmácia, Centro de Ciências da Saúde, Universidade de Caxias do Sul, Rua Francisco Getúlio Vargas, 1130, 95070-560 \\ Caxias do Sul - RS, Brasil \\ Kellen Cristhinia Borges de Souza* \\ Departamento de Ciências Básicas e da Saúde, Universidade Federal de Ciências da Saúde de Porto Alegre, Rua Sarmento Leite, \\ 245, 90050-170 Porto Alegre - RS, Brasil
}

Recebido em 8/8/11; aceito em 28/11/11; publicado na web em 23/1/12

\begin{abstract}
DEVELOPMENT AND VALIDATION OF ANALYTICAL METHOD FOR DETERMINATION OF TIBOLONE IN CAPSULES BY HIGH PERFORMANCE LIQUID CHROMATOGRAPHY. Tibolone is a synthetic steroid used for prevention of bone loss and treatment of menopause symptoms. This article describes the development and validation of an analytical method to quantify tibolone in capsules using high performance liquid chromatography with UV detection. After chromatography conditions are established the validation parameters evaluated were specificity, linearity, precision, accuracy, detection and quantification limits and robustness. The method developed is effective to analyze tibolone in capsules, being able to be used in quality control laboratory routine.
\end{abstract}

Keywords: tibolone; high performance liquid chromatography; validation analytical method.

\section{INTRODUÇÃO}

A tibolona, 7-alfa-metil-17-alfa-etinilestradiol (Figura 1), é um esteroide sintético utilizado na prevenção da perda óssea e tratamento de sintomas do climatério, período caracterizado por uma diminuição gradual na função ovariana. Seu mecanismo de ação ocorre tanto em receptores estrogênicos quanto em progestogênicos e androgênicos, através da ação seletiva de seus diferentes metabólitos sobre cada tecido. $^{1-3}$

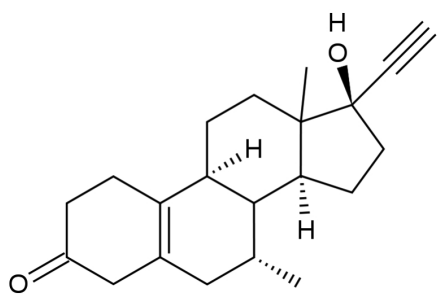

Figura 1. Estrutura química da tibolona

Devido à sua ação específica e à eficácia no aumento da densidade mineral óssea em mulheres na pós-menopausa, a tibolona torna-se uma alternativa de tratamento para mulheres que já não se encontram no período fértil. ${ }^{2}$ Entretanto, devido ao custo significante deste medicamento para a maior parte da população brasileira, a procura por formulações preparadas em Farmácias de Manipulação torna-se uma alternativa viável para a manutenção do tratamento. ${ }^{4,5}$

Por oferecer ampla diversificação de produtos, este setor tem um grande desafio na produção de formulações com garantia de eficácia terapêutica e segurança do paciente. Desta forma, é essencial o controle de qualidade do produto acabado nas farmácias de manipulação, pois um dos parâmetros que mais comumente

*e-mail: kellens@ufcspa.edu.br apresentam desvios da qualidade é a quantidade de fármaco determinada no doseamento. ${ }^{6,7}$

Além disso, a partir do estabelecimento da Resolução da Diretoria Colegiada (RDC) n 33, em 2001, as Farmácias que manipulam formas farmacêuticas passaram a realizar ou terceirizar os ensaios de controle de qualidade dos insumos e dos produtos acabados e, em 2007, com a publicação da $\mathrm{RDC} \mathrm{n}^{\circ} 67$, a prioridade para a realização destes ensaios se concentrou em fármacos cuja dosagem é igual ou inferior a $5 \mathrm{mg}$; desta forma, é essencial o desenvolvimento e o domínio de uma técnica analítica para determinação destes fármacos, entre eles a tibolona, em formulações obtidas em farmácias de manipulação. ${ }^{8,9}$

$\mathrm{Na}$ literatura foram encontrados estudos que empregam métodos para o doseamento de tibolona e metabólitos em fluidos biológicos e ensaios de dissolução. ${ }^{10-13}$ Foram encontradas monografias em compêndios oficiais com métodos para a determinação do teor deste fármaco em matérias-primas e comprimidos. ${ }^{14}$ Embora seja possível a transposição destes métodos para a análise de cápsulas, não existe estudo específico sobre a análise desta forma farmacêutica, que pode apresentar matrizes diferentes de outros formas sólidas. Considerando os aspectos mencionados, o presente trabalho teve por objetivo desenvolver e validar método para a determinação de tibolona em cápsulas, utilizando cromatografia líquida de alta eficiência (CLAE) com detecção no UV.

\section{PARTE EXPERIMENTAL}

\section{Reagentes e padrões}

Utilizou-se como substância química de referência (SQR) tibolona (Sigma Aldrich, Saint Louis, EUA), com pureza de 99,46\%. Foram empregadas cápsulas provenientes de Farmácia de Manipulação, contendo 2,5 mg de tibolona, e matéria-prima (DEG) com teor de $100,37 \%$. Os excipientes empregados foram fosfato de cálcio tribásico, sulfato de cálcio di-hidratado, estearato de magnésio, 
hidroxipropilmetilcelulose, lauril sulfato de sódio e lactose. Foram empregados metanol, acetonitrila e ácido fosfórico com grau HPLC (Merck, Darmstadt, Alemanha) e água ultrapura obtida a partir de sistema de purificação de água Milli-Q Gradient ${ }^{\circledR}$ (Millipore, Bedford, EUA).

\section{Ensaios de solubilidade e análise do espectro de absorção no ultravioleta}

Testou-se a solubilidade da tibolona matéria-prima em água, etanol, metanol e metanol $77 \%$ (v/v), utilizando-se uma quantidade analiticamente pesada com adição gradual dos solventes. Em seguida, realizou-se varredura de absorção no ultravioleta em espectrofotômetro Genesys 10 UV (Madison, EUA), para determinação dos máximos e mínimos de absorção.

\section{Equipamentos e condições cromatográficas}

Foi utilizado um sistema de cromatografia líquida de alta eficiência constituído de bomba LC-10AT (Shimadzu, Kyoto, Japão), detector de arranjo de diodos modelo SPD-M10A, autoinjetor SIL-10AF, degaseficador DGU-20A e um misturador FCV-10AL gerenciado por um controlador SCL-10A, conectado diretamente a um computador equipado com software Shimadzu Class-VP Versão 6.13 SP 2.

Empregou-se uma coluna Agilent (Santa Clara, EUA) Eclipse XDB-C8 de $25 \mathrm{~cm}$ de comprimento, 4,6 $\mathrm{mm}$ de diâmetro e $5 \mu \mathrm{m}$ de tamanho de partícula. Foi empregada fase móvel constituída de acetonitrila e água $(65: 35$, v/v), filtrada em membrana de poliamida (Sartorius, Goettingen, Alemanha) com $0,45 \mu \mathrm{m}$ de porosidade. A vazão da fase móvel foi de $1,0 \mathrm{~mL} \mathrm{~min}^{-1}$. A amostra foi filtrada em membrana de fluoreto de polivinilideno PVDF (Millipore) de 0,45 $\mu \mathrm{m}$ de porosidade. $\mathrm{O}$ volume injetado de amostra foi de $50 \mu \mathrm{L}$ e a detecção foi realizada em comprimento de onda de $204 \mathrm{~nm}$.

\section{Preparação da solução padrão}

Pesou-se $25 \mathrm{mg}$ de tibolona SQR e transferiu-se para balão volumétrico de $100 \mathrm{~mL}$ e adicionou-se aproximadamente $70 \mathrm{~mL}$ de metanol 80\% (v/v), agitando-se manualmente até dispersão do pó e deixando-o em repouso até a dissolução completa. Após este procedimento completou-se o volume com o mesmo solvente obtendo-se uma concentração final de $0,25 \mathrm{mg} \mathrm{mL}^{-1}$.

\section{Preparação da solução amostra - matéria-prima}

Pesou-se $25 \mathrm{mg}$ de tibolona matéria-prima e transferiu-se para balão volumétrico de $100 \mathrm{~mL}$ e adicionou-se aproximadamente 70 $\mathrm{mL}$ de metanol $80 \%$ (v/v), agitando-se manualmente até dispersão do pó e deixando-o em repouso até a dissolução completa. Após este procedimento completou-se o volume com o mesmo solvente e transferiu-se uma alíquota de $4 \mathrm{~mL}$ para balão volumétrico de $10 \mathrm{~mL}$, completando-se o volume com metanol $80 \%$ (v/v), culminando na concentração final de $0,1 \mathrm{mg} \mathrm{mL}^{-1}$. Esta solução foi utilizada somente para o desenvolvimento do método.

\section{Preparação da solução amostra - cápsulas}

Misturou-se o conteúdo de 20 cápsulas e pesou-se quantidade equivalente a $10 \mathrm{mg}$ de tibolona que foram acrescidos de cerca de $40 \mathrm{~mL}$ de metanol $80 \%$ (v/v), em balão volumétrico de $100 \mathrm{~mL}$, e submetidos à agitação mecânica, por $30 \mathrm{~min}$. Ao final deste processo completou-se o volume com o mesmo solvente obtendo-se a concentração final de $0,1 \mathrm{mg} \mathrm{mL}^{-1}$.

\section{Validação do método}

O método proposto foi avaliado considerando os seguintes parâmetros: linearidade, precisão intra- e interdia, exatidão, especificidade, determinação dos limites de detecção e quantificação e robustez. ${ }^{15}$

\section{Especificidade}

Verificou-se a especificidade do método através da análise da matriz de excipientes que compõem a amostra de cápsulas. Para constatar a ausência de interferência no sinal de detecção e a formação de picos da tibolona interpretou-se o seu cromatograma no tempo de retenção referente ao pico da amostra, comparando-o com o cromatograma da SQR.

\section{Linearidade}

A partir da solução padrão de concentração de $0,25 \mathrm{mg} \mathrm{mL}^{-1}$ preparou-se uma curva padrão com 5 concentrações, compreendidas entre 0,05 e $0,15 \mathrm{mg} \mathrm{mL}^{-1}$. Cada concentração foi preparada em triplicata e injetou-se duas vezes cada uma das réplicas.

\section{Precisão}

A precisão intradia foi avaliada em 3 dias diferentes, através da análise em triplicata das concentrações equivalentes a 80,100 e $120 \%$ da concentração teórica do método, com duas injeções por cada réplica, a fim de se avaliar a proximidade dos resultados obtidos, calculados através de curva padrão, em determinados intervalos de tempo. Os resultados obtidos nos diferentes níveis de concentração, para os três dias de análise do ensaio de precisão foram comparados pelo teste da análise de variância (ANOVA) de duas vias com repetições.

\section{Exatidão}

A exatidão foi avaliada através da análise em triplicata, com duas injeções de cada réplica, de uma solução amostra placebo, acrescida de tibolona SQR até as concentrações equivalentes a 80, 100 e $120 \%$ da concentração teórica do teste. A partir disto calculou-se a eficiência do método em quantificar a concentração de SQR adicionada, levando-se em conta a pureza da tibolona adicionada.

\section{Limites de detecção e quantificação}

Os limites de detecção e quantificação foram calculados a partir dos desvios padrões do intercepto e da média do coeficiente angular de três curvas padrões

\section{Robustez}

A robustez do método foi avaliada através de variações deliberadas nos fatores considerados críticos para quantificação de tibolona - pH da água da fase móvel, proporção entre fase orgânica e aquosa na fase móvel, teor de metanol e tempo de agitação empregado na preparação da amostra. Os fatores foram avaliados utilizando desenho fatorial fracionado - Placket-Burman - para 7 fatores e 8 experimentos, sendo incluídos 3 fatores dummy para completar o experimento. Os fatores dummy não causam variações no método nos diferentes níveis e, por isso, podem ser considerados como estimativa do erro. Os efeitos dos fatores foram avaliados $(\alpha$ $=0,05$ ) pelo teste $\mathrm{t}$ Student empregando como estimativa do erro a variação observada na precisão intermediária, uma vez que apenas 3 fatores dummy para estimativa do erro resultam em apenas 3 graus de liberdade para o erro, o que reduz a sensibilidade do teste estatístico. ${ }^{16-18}$

As amostras foram preparadas conforme descrito anteriormente empregando as variações apresentadas na Tabela 1 e o desenho experimental selecionado apresentado na Tabela 2 . 
Tabela 1. Fatores e níveis analisados no ensaio de robustez do método de doseamento de tibolona em cápsulas por cromatografia líquida de alta eficiência

\begin{tabular}{lcccc}
\hline Fatores & Nominal & Limites & $(-)$ & $(+)$ \\
\hline pH água da FM & 6,0 & 1,0 & 5,0 & 7,0 \\
Porção orgânica da FM & 65,0 & 2,0 & 63,0 & 67,0 \\
Teor de metanol utilizado & 80,0 & 5,0 & 75,0 & 85,0 \\
Tempo de agitação & 30,0 & 5,0 & 25,0 & 35,0 \\
Dummy & Amostra & & & \\
\hline
\end{tabular}

FM = fase móvel; Dummy $=$ fator sem significado físico, utilizado para completar o desenho fatorial.

\section{RESULTADOS E DISCUSSÃO}

O desenvolvimento de método de análise de hormônios em formas farmacêuticas torna-se importante, uma vez que muitos deles são fármacos de baixa dosagem, na qual a probabilidade de erro durante a manipulação é maior. Em geral, são fármacos de difícil quantificação por métodos menos sofisticados que a CLAE.

Em estudo realizado com a tibolona em amostras de plasma e tecidos corporais, empregando cromatografia líquida acoplada à espectrometria de massa, foram obtidos resultados satisfatórios que possibilitaram cálculos de parâmetros farmacocinéticos do fármaco e seus metabólitos. ${ }^{19}$

Outro estudo empregou, na análise de tibolona e seus metabólitos por CLAE, uma coluna Symmetry Shield RP 18 (3,5 $\mu \mathrm{m}$ x 3,0 mm x $150 \mathrm{~mm}$ ), fase móvel com eluição gradiente composta de ácido trifluoroacético $0,1 \%$ (v/v) em água e ácido trifluoroacético $0,1 \%$ (v/v) em acetonitrila, com fluxo de $0,5 \mathrm{~mL} \mathrm{~min}^{-1}$, volume de injeção de $20 \mu \mathrm{L}$ e detecção em $210 \mathrm{~nm}$. Através deste método foi possível a análise de produtos de reação enzimática entre a tibolona e as sulfotransferases intestinais de mulheres na pós-menopausa. ${ }^{20}$

Alternativamente, determinou-se o metabolismo da tibolona em amostras de plasma, urina e fezes empregando uma coluna $\mu$ Bondpak $\mathrm{C} 18(3,9 \times 300 \mathrm{~mm})$ a $50{ }^{\circ} \mathrm{C}$, com fluxo de $1,7 \mathrm{~mL} \mathrm{~min}^{-1}$, fase móvel composta de acetato de amônio $0,1 \mathrm{M} \mathrm{pH}$ 4,2 e metanol, e detecção em $254 \mathrm{~nm}{ }^{1}$

Também foi possível definir o perfil de dissolução de formas farmacêuticas de liberação imediata, empregando lauril sulfato de sódio como meio de dissolução através do uso de uma coluna Hipersil ${ }^{\circledR}$ ODS $(5 \mu \mathrm{m} \times 4,6 \mathrm{~mm} \times 100 \mathrm{~mm})$ a $40{ }^{\circ} \mathrm{C}$, fase móvel de metanol e água

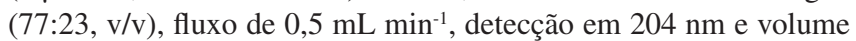
de injeção de $200 \mu \mathrm{L}$. $^{21}$

Já para a análise de tibolona em diferentes adsorbatos, foi desenvolvido método composto por uma coluna CC 250/4 Nucleosil 100-5

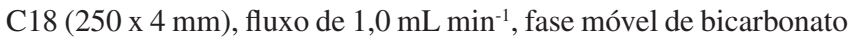

de amônio $0,005 \%$ (v/v) em água e acetonitrila (50:50, v/v) e detecção em $210 \mathrm{~nm}$, caracterizando-os quanto à concentração do fármaco..$^{22,23}$

Além destes trabalhos, são descritos mais dois métodos de quantificação de tibolona por titulometria e por CLAE. O primeiro método utiliza uma reação entre a tibolona e o nitrato de prata, seguida de titulação com solução de hidróxido de sódio. Já o segundo, sugere o uso de uma coluna $\mathrm{C} 18(15 \mathrm{~cm}$ x $3,9 \mathrm{~mm} \times 4 \mu \mathrm{m})$ a $40{ }^{\circ} \mathrm{C}$, fase

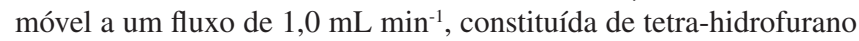
e água (28:72, v/v), detecção em $240 \mathrm{~nm}$ após injeção de $10 \mu \mathrm{L}$. Através destas técnicas é possível determinar o teor de tibolona em matérias-primas e em comprimidos, respectivamente. ${ }^{14}$

Com base nos trabalhos citados, foi realizado o desenvolvimento e otimização do método de doseamento de tibolona em cápsulas por CLAE.

\section{Desenvolvimento e otimização do método}

Com objetivo de selecionar o melhor solvente para o estudo verificou-se a solubilidade da tibolona matéria-prima, constatando-se que o fármaco é completamente solúvel em metanol, solúvel em etanol e metanol 77\% (v/v) e praticamente insolúvel em água.

A amostra, solubilizada em metanol, foi submetida à análise por espectrofotometria no ultravioleta para determinação do comprimento de onda de detecção. Alguns métodos descritos na literatura descrevem o emprego de comprimentos de onda de detecção de 210 e $254 \mathrm{~nm}$, entretanto, para este estudo, o comprimento de onda de $204 \mathrm{~nm}$ foi o escolhido por ser o ponto máximo de absorção no espectro obtido para a tibolona. Com base nisto decidiu-se realizar a análise por cromatografia líquida de alta eficiência, uma vez que a amostra apresenta absorção em comprimentos de onda baixos, onde a interferência dos excipientes utilizados se torna mais provável. ${ }^{24-26}$

Para a definição das condições cromatográficas, foi realizada análise empregando-se a solução amostra - matéria-prima. Utilizouse, inicialmente, fase móvel composta por metanol e água (77:23), empregou-se metanol como diluente, coluna C18 de $15 \mathrm{~cm}$ x 4,6 mm x $5 \mu \mathrm{m}$ (Shimadzu), volume de injeção de $20 \mu \mathrm{L}$, fluxo de $1,0 \mathrm{~mL}$ min $^{-1}$ e detecção em $204 \mathrm{~nm}$. Entretanto, foram observados problemas de estabilização de pressão com a coluna em questão.

Posteriormente, foi empregada uma coluna Phenomenex C18 de $25 \mathrm{~cm}$ x 4,6 mm x $5 \mu \mathrm{m}$ e obteve-se um resultado reprodutível, mas com problemas no formato do pico, que apresentou assimetria $(1,06) \mathrm{e}$ fator de capacidade $(1,37)$ satisfatórios e resolução $(0,59)$ insatisfatória. Como alternativa utilizou-se fase móvel como diluente, entretanto não foi possível obter melhora significativa no formato do pico.

Com o objetivo de obter melhor definição do pico cromatográfico foram testadas diferentes proporções de fase móvel, volumes de injeção e soluções diluentes, verificando-se melhores resultados com

Tabela 2. Desenho experimental - Placket-Burman - selecionado para avaliação da robustez do método de doseamento de tibolona em cápsulas por cromatografia líquida de alta eficiência

\begin{tabular}{|c|c|c|c|c|c|c|c|}
\hline $\operatorname{Exp} n^{\circ}$ & Teor de metanol & Tempo agitação & Dummy & Fase orgânica na FM & Dummy & $\mathrm{pH}$ da água da FM & Dummy \\
\hline 1 & $(+)$ & $(+)$ & $(+)$ & $(-)$ & $(+)$ & $(-)$ & $(-)$ \\
\hline 2 & $(-)$ & $(+)$ & $(+)$ & $(+)$ & $(-)$ & $(+)$ & $(-)$ \\
\hline 3 & $(-)$ & $(-)$ & $(+)$ & $(+)$ & $(+)$ & $(-)$ & $(+)$ \\
\hline 4 & $(+)$ & $(-)$ & $(-)$ & $(+)$ & $(+)$ & $(+)$ & $(-)$ \\
\hline 5 & $(-)$ & $(+)$ & $(-)$ & $(-)$ & $(+)$ & $(+)$ & $(+)$ \\
\hline 6 & $(+)$ & $(-)$ & $(+)$ & $(-)$ & $(-)$ & $(+)$ & $(+)$ \\
\hline 7 & $(+)$ & $(+)$ & $(-)$ & $(+)$ & $(-)$ & $(-)$ & $(+)$ \\
\hline 8 & $(-)$ & $(-)$ & $(-)$ & $(-)$ & $(-)$ & $(-)$ & $(-)$ \\
\hline
\end{tabular}

FM = fase móvel; Dummy $=$ fator sem significado físico utilizado para completar o desenho fatorial. 
a fase móvel composta por metanol e água 65:35 (v/v), volume de injeção de $100 \mu \mathrm{L}$, fluxo de $1,5 \mathrm{~mL} \mathrm{~min}^{-1}$ e empregando-se metanol $80 \%$ como solução diluente. Nestas condições cromatográficas foi possível obter fator capacidade de 3,54 , assimetria de 1,05 , resolução de 19,2 e tempo de retenção para tibolona de 14,2 min.

Com a finalidade de estabilizar a linha de base adicionou-se à fase móvel selecionada diferentes proporções de acetonitrila; obteve-se o tempo de retenção da amostra de 13,8 min e maior proximidade entre os picos correspondentes aos solventes em tempos de retenção menores. O sistema de eluição que proporcionou melhor estabilidade da linha de base foi metanol, água e acetonitrila $(57: 8: 35 \mathrm{v} / \mathrm{v} / \mathrm{v})$.

Na tentativa de reduzir o tempo de análise foi testada a condição cromatográfica composta por coluna de sílica ligada a octilsilano, com $25 \mathrm{~cm}$ de comprimento, 4,6 mm de diâmetro e $5 \mu \mathrm{m}$ de tamanho de partícula, fluxo de 1,0 mL min ${ }^{-1} \mathrm{e}$ fase móvel composta de acetonitrila e água (65:35 v/v). Obteve-se um cromatograma satisfatório, com boa resolução do pico cromatográfico $(6,67)$, assimetria $(1,16)$, fator capacidade $(1,87)$, tempo de retenção de $6,61 \mathrm{~min}$, linha de base estável e menor volume de injeção $(50 \mu \mathrm{L})$.

\section{Validação do método}

O desenvolvimento de um método analítico e a adaptação de método conhecido envolve um processo de avaliação que estime sua eficiência na rotina do laboratório. Devido a isto, a validação de um método é um meio eficaz para garantir a confiança necessária no resultado emitido pelo método em questão durante uma análise de rotina, principalmente no que diz respeito à reprovação de amostras.

No presente estudo, o método é destinado à quantificação do componente ativo em uma matriz; desta forma, os parâmetros recomendados para serem avaliados em um ensaio de validação são especificidade, intervalo de trabalho, linearidade, precisão e exatidão, sendo facultativa a análise de outros parâmetros. ${ }^{27}$

\section{Especificidade}

A especificidade foi avaliada através da preparação de amostras contendo somente os excipientes utilizados na preparação das cápsulas em comparação com o padrão. Após análise dos cromatogramas, constatou-se que os excipientes não interferem na detecção da tibolona (Figura 2) uma vez que não há sinal de pico no cromatograma de excipientes no tempo de retenção correspondente ao fármaco e a pureza do pico obtido para a tibolona foi igual a 1,0.

\section{Linearidade}

A análise do parâmetro linearidade fornece informações sobre a capacidade do método em fornecer resultados proporcionais à concentração do analito presente na amostra. A linearidade foi avaliada calculando-se a regressão linear pelo método dos quadrados mínimos, utilizando-se uma curva composta por 5 concentrações, em triplicata, dentro do intervalo de concentrações estabelecido para o padrão (0,050-0,150 $\left.\mathrm{mg} \mathrm{mL}^{-1}\right)$.

Foi obtida reta de regressão $y=36335184 x+228655$, onde $y$ representa a área do pico (mAu.s) e x a concentração de tibolona (mg $\left.\mathrm{mL}^{-1}\right)$. O coeficiente de correlação (r) foi de 0,9998 , estando em conformidade com o critério de aceitação. ${ }^{18}$ Não se verificou a presença de valores atípicos nos resíduos padronizados (todos os resultados entre -2,0 e 2,0) ou tendências nas análises dos resíduos, mostrando boa adequação dos resultados ao modelo de primeiro grau proposto.

\section{Precisão}

A precisão tem por finalidade comprovar a capacidade do método de proporcionar o mesmo resultado para a mesma amostra em
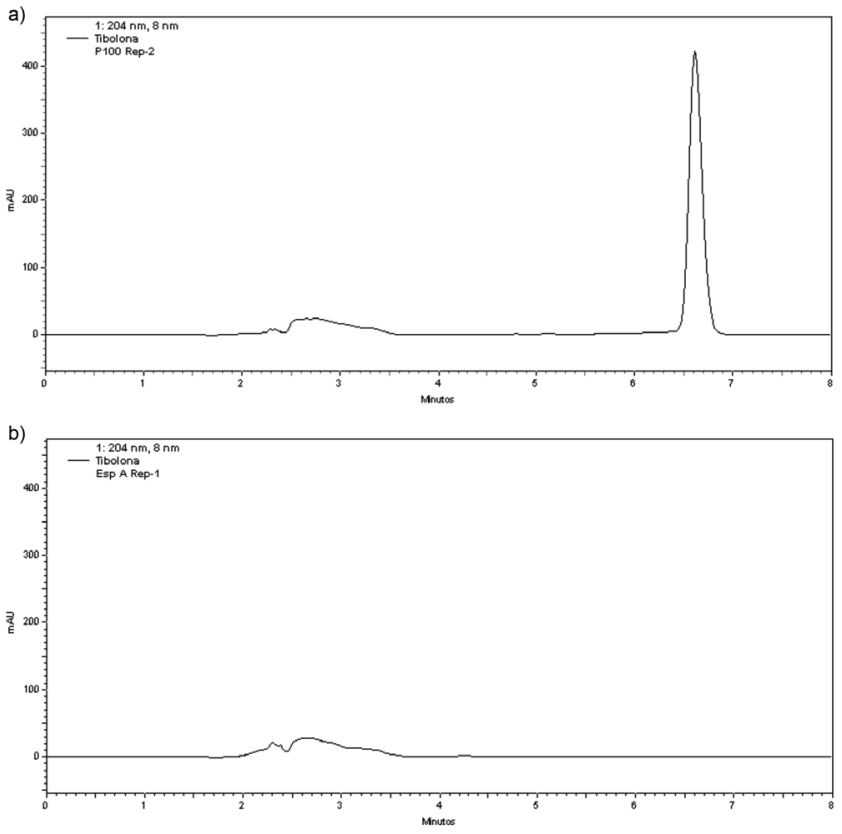

Figura 2. Cromatogramas obtidos por CLAE para o padrão de tibolona na concentração de 0,1 $\mathrm{mg} \mathrm{mL}^{-1}$ (a) e para a matriz de excipientes (b)

diferentes intervalos de tempo.. Os resultados, dos três dias de análise, encontram-se na Tabela 3.

Tabela 3. Resultados obtidos em três dias de análise para as concentrações equivalentes a 80,100 e $120 \%$ da concentração teórica da amostra, no ensaio de avaliação da precisão do método de doseamento de tibolona em cápsulas por cromatografia líquida de alta eficiência

\begin{tabular}{cccccc}
\hline Concentração & Dia 1 & Dia 2 & Dia 3 & Média & DPR \\
\hline \multirow{2}{*}{$80 \%$} & $100,0 \%$ & $102,0 \%$ & $103,7 \%$ & & \\
& $101,5 \%$ & $102,7 \%$ & $101,2 \%$ & $101,5 \%$ & $1,1 \%$ \\
& $101,4 \%$ & $100,7 \%$ & $100,5 \%$ & & \\
\hline \multirow{2}{*}{$100 \%$} & $100,4 \%$ & $101,0 \%$ & $102,2 \%$ & & \\
& $103,1 \%$ & $102,5 \%$ & $103,4 \%$ & $101,7 \%$ & $1,1 \%$ \\
& $101,4 \%$ & $100,9 \%$ & $100,3 \%$ & & \\
\hline \multirow{2}{*}{$120 \%$} & $100,2 \%$ & $103,0 \%$ & $103,9 \%$ & & \\
& $102,8 \%$ & $100,0 \%$ & $103,9 \%$ & $101,9 \%$ & $1,7 \%$ \\
\hline Média & $101,1 \%$ & $99,5 \%$ & $102,6 \%$ & & \\
DPR & $101,3 \%$ & $101,4 \%$ & $102,4 \%$ & Total & $101,7 \%$ \\
\hline
\end{tabular}

$\mathrm{DPR}=$ desvio padrão relativo

Na Tabela 4 são apresentados os resultados da ANOVA, além dos parâmetros que resumem o teste de precisão (repetibilidade e precisão intermediária). A variância do erro experimental (resíduo ou variação dentro dos tratamentos), no ensaio da ANOVA, pode ser associada à repetibilidade através da Equação $1 .{ }^{28}$

$$
R p=\frac{\sqrt{Q M E}}{\bar{x}} \times 100 \%
$$

$\mathrm{Rp}=$ repetibilidade; $\mathrm{QME}=$ quadrado médio do erro experimental obtido pela ANOVA; $\overline{\mathrm{x}}=$ média dos resultados do doseamento para as amostras no ensaio de precisão. 
Tabela 4. Resultados da ANOVA obtidos para os parâmetros de repetibilidade e precisão intermediária do método de doseamento de tibolona em cápsulas por cromatografia líquida de alta eficiência

\begin{tabular}{ccccccc}
\hline Fonte da variação & SQ & gl & QM & F & valor-P & F crítico \\
\hline Níveis & $5,82 \mathrm{E}-05$ & 2 & $2,91 \mathrm{E}-05$ & 0,168 & 0,847 & 3,555 \\
Dias & $6,97 \mathrm{E}-04$ & 2 & $3,48 \mathrm{E}-04$ & 2,011 & 0,163 & 3,555 \\
Interações & $6,63 \mathrm{E}-04$ & 4 & $1,66 \mathrm{E}-04$ & 0,957 & & \\
Erro & $3,12 \mathrm{E}-03$ & 18 & $1,73 \mathrm{E}-04$ & & & \\
Total & $4,54 \mathrm{E}-03$ & 26 & & & \\
Repetibilidade $=$ & $1,3 \%$ & $0,4 \%$ & & & & \\
Precisão intermediária $=$ & & & & & \\
\hline
\end{tabular}

$\mathrm{SQ}=$ Soma dos quadrados; $\mathrm{gl}$ = Graus de Liberdade; $\mathrm{QM}=$ Quadrado Médio.

A precisão intermediária pode ser verificada através dos resultados da variação entre os dias da ANOVA, porém desta variação deve ser retirada a variância do erro experimental, como mostra a Equação 2. Os resultados de repetibilidade $(1,29 \%)$ e precisão intermediária $(0,43 \%)$ encontram-se dentro dos limites aceitáveis para doseamento de fármacos em cápsulas por CLAE, que em geral é de 2,0\%.26,27

$$
P I=\frac{\sqrt{\frac{Q M D i a s-Q M E}{r}}}{\bar{x}} \times 100 \%
$$

$\mathrm{PI}=$ precisão intermediária; $\mathrm{QME}=$ quadrado médio do erro experimental obtido pela ANOVA; $\overline{\mathrm{x}}=$ média dos resultados do doseamento para as amostras no ensaio de precisão; $r=$ quantidade de valores para cada nível avaliado.

\section{Exatidão}

A exatidão visa a comprovação da eficácia do método na quantificação de determinado analito em uma amostra. Os resultados do ensaio de recuperação das amostras analisadas nos três diferentes níveis de concentração estão apresentados na Tabela 5. Os resultados estão de acordo com os critérios de aceitação estabelecidos, que preconizam exatidão entre 98,0 e 102,0\%, com desvio padrão relativo menor ou igual a 2,0\%, para fármacos em cápsulas com doseamento por CLAE. ${ }^{26}$

Tabela 5. Resultados obtidos em três dias de análise para os diferentes níveis, equivalentes a 80,100 e $120 \%$ da concentração teórica do método $\left(0,1 \mathrm{mg} \mathrm{mL}^{-1}\right)$, no ensaio de avaliação da exatidão do método de doseamento de tibolona em cápsulas por cromatografia líquida de alta eficiência

\begin{tabular}{cccccc}
\hline Níveis & Dia 1 & Dia 2 & Dia 3 & Média & DPR \\
\hline \multirow{5}{*}{$80 \%$} & $101,9 \%$ & $101,4 \%$ & $101,1 \%$ & & \\
& $100,9 \%$ & $101,0 \%$ & $102,1 \%$ & $101,4 \%$ & $0,5 \%$ \\
& $101,7 \%$ & $100,8 \%$ & $101,9 \%$ & & \\
\hline \multirow{5}{*}{$100 \%$} & $99,6 \%$ & $98,8 \%$ & $99,4 \%$ & & \\
& $99,9 \%$ & $100,0 \%$ & $102,1 \%$ & $100,2 \%$ & $1,1 \%$ \\
& $100,00 \%$ & $100,1 \%$ & $101,7 \%$ & & \\
$120 \%$ & $98,6 \%$ & $103,8 \%$ & $98,5 \%$ & & \\
& $99,7 \%$ & $100,1 \%$ & $101,2 \%$ & $100,3 \%$ & $1,7 \%$ \\
\hline Média & $99,2 \%$ & $101,9 \%$ & $99,8 \%$ & & \\
DPR & $100,2 \%$ & $100,9 \%$ & $100,9 \%$ & Total & $100,6 \%$ \\
\hline DPR $=$ desvi & $1,1 \%$ & $1,4 \%$ & $1,3 \%$ & & $1,3 \%$ \\
\hline
\end{tabular}

$\mathrm{DPR}=$ desvio padrão relativo

Limites de detecção e quantificação

Os cálculos dos limites de detecção e quantificação fornecem a comprovação de que o intervalo de concentrações de trabalho escolhido se apresenta capaz de detectar e quantificar o analito desejado. Estimaram-se os valores para limites de detecção e quantificação, respectivamente, de 2,9 e $9,8 \mu \mathrm{g} \mathrm{mL}^{-1}$, podendo-se concluir que o intervalo de trabalho do método $\left(50\right.$ a $\left.150 \mu \mathrm{g} \mathrm{mL}^{-1}\right)$ está em conformidade com os valores calculados.

Robustez

Os resultados da robustez foram avaliados através do teste $\mathrm{t}$ Student, no qual o valor de t calculado foi comparado com o valor de $t$ crítico bicaudal assumindo $\alpha=0,05$. Nesta avaliação são levados em conta os efeitos de cada fator envolvido na análise, observando-se os níveis estabelecidos para cada um e o número de ensaios do desenho experimental elaborado. As relações entre estes parâmetros estão representadas nas Equações 3 e 4.

$$
E_{x}=\frac{\sum Y(+)}{N / 2}-\frac{\sum Y(-)}{N / 2}
$$

$\mathrm{E}_{\mathrm{x}}=$ efeito do fator em análise; $\Sigma \mathrm{Y}(+)$ e $\Sigma \mathrm{Y}(-)=$ soma das respostas onde $\mathrm{Y}$ está nos níveis extremos $(+)$ e $(-)$, respectivamente; $\mathrm{N}=$ número de experimentos do desenho experimental.

$$
t=\frac{E_{x}}{E_{e}}
$$

$\mathrm{E}_{\mathrm{x}}=$ efeito do fator em anális; $\mathrm{E}_{\mathrm{e}}=$ erro experimental do método.

Em todos os fatores testados o valor de t calculado foi inferior ao $t$ crítico (Tabela 6), comprovando que o método é robusto para os parâmetros testados ( $\mathrm{pH}$ da água da fase móvel, proporção entre fase orgânica e aquosa na fase móvel, teor do metanol e tempo de agitação utilizados na preparação da amostra).

Tabela 6. Resultados da influência dos efeitos dos parâmetros testados no

\begin{tabular}{|c|c|c|c|c|}
\hline $\operatorname{Exp~n}^{\circ}$ & $\begin{array}{l}\text { Teor de } \\
\text { metanol }\end{array}$ & $\begin{array}{c}\text { Tempo } \\
\text { agitação }\end{array}$ & $\begin{array}{c}\text { Fase orgânica } \\
\text { na FM }\end{array}$ & $\begin{array}{c}\text { pH da água } \\
\text { da FM }\end{array}$ \\
\hline Efeito (+) & $91,2 \%$ & $91,3 \%$ & $91,2 \%$ & $90,8 \%$ \\
\hline Efeito (-) & $91,3 \%$ & $91,3 \%$ & $91,4 \%$ & $91,7 \%$ \\
\hline Efeito (Ex) & $0,2 \%$ & $0,0 \%$ & $0,2 \%$ & $0,9 \%$ \\
\hline Erro (Ee) & $0,4 \%$ & & & \\
\hline t calculado & $0,37^{\mathrm{a}}$ & $0,00^{\mathrm{a}}$ & $0,51^{\mathrm{a}}$ & $2,04^{a}$ \\
\hline $\mathrm{t}_{(0.05 ; 18)}$ & 2,101 & & & \\
\hline
\end{tabular}
ensaio de robustez do método de doseamento de tibolona em cápsulas por cromatografia líquida de alta eficiência

\section{CONCLUSÃO}

Durante o desenvolvimento do método, constataram-se as diferenças entre o tipo de coluna empregada (octilsilano e octadecilsilano) no tempo de retenção da tibolona e no desenvolvimento do 
cromatograma. Observou-se também que a melhor proporção entre as partes orgânica e aquosa na fase móvel foi de 65:35 (v/v), para ambas as colunas, obtendo-se bons resultados para os parâmetros cromatográficos, como pureza de pico, resolução e assimetria.

Para a parcela orgânica da fase móvel, a acetonitrila apresentou-se como solvente mais adequado, por fornecer estabilidade à linha de base e melhor definição da forma do pico cromatográfico com relação ao emprego do metanol. Já para a porção aquosa, o emprego de água foi eficaz no ajuste da separação do pico do fármaco e não evidenciou uma necessidade do uso de tampões para realização da análise.

Os resultados dos parâmetros de validação avaliados foram correspondentes com os critérios de aceitação utilizados, comprovando que o método é linear no intervalo de trabalho proposto, possui boa precisão e exatidão, além de estar acima dos limites de detecção e quantificação estimados. Além disso, o método demonstrou robustez quando exposto a variações deliberadas nas condições experimentais estabelecidas.

O método desenvolvido apresentou resultados satisfatórios que justificam o seu emprego na rotina de análises em laboratório de controle de qualidade.

\section{REFERÊNCIAS}

1. Vos, R. M. E.; Krebbers, S. F. M.; Verhoeven, C. H. J.; Delbressine, L. P. C.; Drug Metab. Dispos. 2002, 30, 106.

2. Henriques, H. N.; Câmara, N. R.; Carvalho, A. C. B.; Pantaleão, J. A. S.; Guzmán-Silva, M. A.; Rev. Bras. Ginecol. Obstet. 2009, 31, 124.

3. Kloosterboer, H. J.; EMAS 2004, 48, S30.

4. http://www.consultaremedios.com.br, acessada em Novembro 2011.

5. http://www.ibge.gov.br, acessada em Novembro 2011.

6. Polonini, H. C.; Santos, F. C.; Vaz, U. P.; Brandão, M. F.; Raposo, N. R. B.; Ferreira, A. O.; Quim. Nova 2011, 34, 516.

7. Couto, A. G.; Tavares, R. C.; Rev. Ciênc. Farm. Básica Apl. 2011, 32, 263.

8. Brasil, Ministério da Saúde, Agência Nacional de Vigilância Sanitária (ANVISA); Resolução RDC No 33, de 19/04/2000, Regulamento Técnico sobre Boas Práticas de Manipulação de Medicamentos em farmácias e seus Anexos.
9. Brasil, Ministério da Saúde, Agência Nacional de Vigilância Sanitária (ANVISA); Resolução RDC No 67, de 09/10/2007, Dispõe sobre Boas Práticas de Manipulação de Preparações Magistrais e Oficinais para Uso Humano em farmácias.

10. Papadimitriou, S.; Bikiaris, D.; Drug Dev. Ind. Pharm. 2009, 35, 1128.

11. Penning, T. M.; Lee, S. H.; Gutierrez, A.; Blair, I. A.; J. Steroid Biochem. Mol. Biol. 2010, 121, 546.

12. Fekete, S.; Fekete, J.; Ganzler, K. J.; J. Pharm. Biomed. Anal. 2009, 50, 703 .

13. Donald, P.; Anti-Cancer Agents Med. Chem. 2009, 9, 642.

14. Comission, B. P.; British Pharmacopoeia 2011, The Stationery Office: London, 2011.

15. International Conference on Harmonization - ICH; International Conference on Harmonization of Technical Requirements for Registration of Pharmaceuticals for Human Use, Validation of Analytical Procedures: Methodology, 1996.

16. Heyden, Y. V.; Nijhuis, A.; Smeyers-Verbeke, J.; Vandeginste, B. G. M.; Massart, D. L.; J. Pharm. Biomed. Anal. 2001, 24, 723.

17. Paim, C. S.; Gonçalves, H.; Lange, A.; Miron, D.; Steppe, M.; Anal. Lett. 2008, 41, 571

18. Paim, C. S.; Gonçalves, H. M. L.; Miron, D.; Sippel, J.; Steppe, M.; Chromatographia 2007, 65, 595.

19. Verheul, H. A. M.; van-Lersel, M. L. P. S.; Delbressine, L. P. C.; Kloosterboer, H. J.; Drug Metab. Dispos. 2007, 35, 1105.

20. Wang, M.; Ebmeier, C. C.; Olin, J. R.; Anderson, R. J.; Steroids 2006, 71,343 .

21. De Haan, P.; US pat 0,134,191 2006.

22. Glaenzer, K.; US pat $0,176,6792005$

23. Glaenzer, K.; US pat 0,003,977 2006.

24. Favoretto, L. B.; Souza, J. M. O.; Bonfilio, R.; Azevedo, R. C. P.; Araújo, M. B.; Quim. Nova 2010, 33, 1585.

25. Frazon, M. A.; Silvestrin, M. S.; Visão Acadêmica 2010, 11, 59.

26. Oliveira, D. M.; Markman, B. E. O.; Uessugui, O.; Wu, E. M.; Magnelli, R. F.; Rev. Ciênc. Farm. Básica Apl. 2010, 31, 211.

27. Shabir, G. A.; J. Chromatogr., A 2003, 987, 57.

28. Ermer, J.; Ploss, H.; J. Pharm. Biomed. Anal. 2005, 37, 859. 